\title{
(C) OPEN ACCESS \\ Clinical significance of atrial high-rate episodes for thromboembolic events in Japanese population
}

\author{
Hiroshi Kawakami, ${ }^{1}$ Takayuki Nagai, ${ }^{1}$ Makoto Saito, ${ }^{2}$ Shinji Inaba, ${ }^{2}$ Fumiyasu Seike, \\ Kazuhisa Nishimura, ${ }^{1}$ Katsuji Inoue, ${ }^{1}$ Takafumi Okura, ${ }^{1}$ Takumi Sumimoto, ${ }^{2}$ \\ Shigeki Uemura, ${ }^{3}$ Jitsuo Higaki, ${ }^{1}$ Shuntaro Ikeda ${ }^{1}$
}

'Department of Cardiology, Pulmonology, Hypertension, and Nephrology, Ehime University Graduate School of Medicine, Toon, Ehime, Japan

${ }^{2}$ Division of Cardiology, Kitaishikai Hospital, Ehime, Japan

${ }^{3}$ Division of Cardiology, Yawatahama City General Hospital, Ehime, Japan

\section{Correspondence to} Dr Takayuki Nagai, Department of Cardiology, Pulmonology, Hypertension, and Nephrology, Ehime University Graduate School of Medicine, Toon, Ehime 791-0295, Japan; nagait@m. ehime-u.ac.jp

Received 1 August 2017 Revised 10 October 2017 Accepted 23 October 2017
CrossMark

To cite: Kawakami $\mathrm{H}$, Nagai T, Saito M

et al. Heart Asia

2017;9:1-6. doi:10.1136/

heartasia-2017-010954

\section{ABSTRACT}

Objective The clinical significance of atrial high-rate episodes (AHREs) detected by cardiac devices among patients with implantable pacemakers has recently emerged. However, the relationship between AHREs and ischaemic stroke and systemic embolism (SE) is not well understood in the Japanese population.

Methods This study included 343 patients with pacemakers capable of continuous atrial rhythm monitoring (167 males; mean age, $80 \pm 7$ years). Atrial tachyarrhythmia detection was programmed to the nominal setting of each device, and AHRE was defined as any episode of sustained atrial tachyarrhythmia lasting for more than 6 min. Thromboembolic risk was defined based on the $\mathrm{CHADS}_{2}$ score.

Results During the follow-up period ( $52 \pm 30$ months), $165(48 \%)$ patients had at least one episode of AHREs, and $19(6 \%)$ patients experienced stroke/SE. Among patients who experienced stroke/SE, 14 had AHREs before the stroke/SE. AHREs were significantly associated with stroke/SE (HR 2.87; 95\% Cl 1.10 to 8.90; $p=0.03$ ). Subgroup analysis conducted to investigate the impact of the CHADS 2 score severity on stroke/SE revealed that AHREs were not associated with stroke/SE in patients with low or intermediate thromboembolic risk (CHADS, score $0-2 ; n=217$ ). In contrast, among patients with high thromboembolic risk (CHADS $_{2}$ score $>2 ; n=126)$, there was a significant association between AHREs and the incidence of stroke/SE (HR 3.73; $95 \% \mathrm{Cl} 1.06$ to $13.1 ; \mathrm{p}=0.04)$.

Conclusion AHREs detected by pacemaker were associated with ischaemic stroke/SE in the Japanese population. However, this association was observed only in the high thromboembolic risk group.

\section{INTRODUCTION}

The incidence of atrial fibrillation (AF), which is the most common arrhythmia with clinical significance among elderly populations, is increasing worldwide. ${ }^{1}$ As $\mathrm{AF}$ is a well-established risk factor for thromboembolic events including ischaemic stroke, ${ }^{2}$ anticoagulation therapy is recommended based on $\mathrm{CHADS}_{2}$ and $\mathrm{CHA}_{2} \mathrm{DS}_{2}-$ VASc scores in patients with non-valvular $\mathrm{AF}^{3-5}$

The clinical significance of AF detected by cardiac implantable electronic devices, which are recorded as atrial high-rate episodes (AHREs), has emerged recently. ${ }^{67}$ However, due to the controversial relationship between AHREs and thromboembolic events, which has not been extensively studied in Japanese populations, appropriate anticoagulation therapy strategies for the management of AHREs have not been established.

The aim of this study was to investigate the impact of AHREs on ischaemic stroke in the Japanese population.

\section{METHODOLOGY}

\section{Study population and design}

This was a multicentre retrospective cohort study. The study population included patients from Ehime University Hospital, Kitaishikai Hospital and Yawatahama City General Hospital in Japan. Patients who were 65 years of age or older and underwent their first implantation with a Medtronic dual-chamber pacemaker for sinus node disease or atrioventricular block between January 2005 and December 2014 were included. Patients with prior paroxysmal AF were included, but those with persistent or permanent $\mathrm{AF}$ at the time of pacemaker implantation were excluded from this study because almost all these patients underwent implantation with single-chamber pacemakers (right ventricular lead only). Among a total of 392 patients who were identified, 49 patients were excluded due to the lack of details on the device data due to loss to follow-up, terminal illnesses limiting survival at implantation, atrial lead failure or placement of replacement devices due to infection. Therefore, 343 patients were included in the final analysis (figure 1). All patients underwent follow-up evaluations at each hospital, including clinical and device checks at least every 3-6 months. The strategy for overall medication strategy and the medical treatment for embolism prevention was determined by the physicians attending the outpatient clinic at each institute. The study protocol was approved by the ethics committees of all participating institutions (IRB No. 1201012; 23 January 2017).

\section{Clinical parameters}

Clinical parameters were retrospectively collected from the medical records. Information gathered from the records included the reason for pacemaker implantation, presence of comorbidities, medication usage, echocardiographic data and previously documented AF. Risk factors for thromboembolic events were assessed at baseline to classify patients according to the $\mathrm{CHADS}_{2}$ score. ${ }^{8}$

\section{Implanted devices and detection of AHREs}

All patients received pacemakers based on a class I or IIa indication for cardiac pacemaker set by the Japanese Circulation Society, ${ }^{9}$ and all 


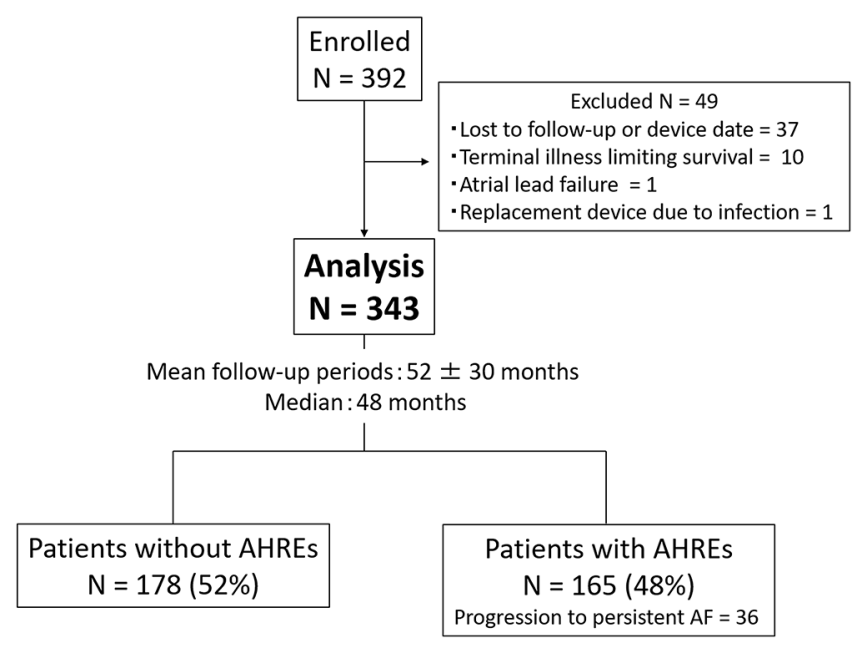

Figure 1 Assembly of the cohort and the results of continuous monitoring by pacemaker during the follow-up period. AF, atrial fibrillation; AHRE, atrial high-rate episode.

implanted cardiac devices were Medtronic dual-chamber pacemakers (Sensia DR, Adapta DR, EnPulse2 DR, Kappa 700 DR, Kappa 900 DR, EnRhythm DR, Advisa DR MRI, Advisa DR; Medtronic, Minneapolis, Minnesota, USA). We enrolled only patients with Medtronic pacemaker implantation to unify the detection methods of AHREs in this study. Pacemakers were programmed to dual-chamber operation with active mode switching, and atrial tachyarrhythmia detection was programmed to the nominal setting of each device. A rate of 175 beats per minute was used to detect atrial arrhythmia in this study. AHRE was defined as any episode of sustained atrial tachyarrhythmia lasting for more than 6 min according to a previous study. ${ }^{10} \mathrm{AF}$ episodes lasting longer than 7 days or those requiring chemical or electrical cardioversion were referred to as persistent AF according to the 2012 hours/EHRA/ECAS expert consensus statement. ${ }^{11}$ Details of device-detected atrial tachyarrhythmia of any duration or atrial morphology were documented during all follow-up periods.

\section{Definition of thromboembolic events}

The primary endpoint of this study was the incidence of ischaemic stroke or systemic embolism (SE), which was determined by the review of medical records performed by an independent and blinded physician. Ischaemic stroke was defined as a neurological deficit with sudden onset persisting for more than 24 hours. CT was performed in all patients who were suspected to have suffered a stroke. Patients with intracranial haemorrhages were excluded. Transient ischaemic attack events were also excluded in this study to rule out false-positive cases.

\section{Statistical analysis}

Overall, $<2 \%$ of observations were missing, and continuous variables were imputed using the corresponding mean values. Continuous variables were expressed as means $\pm S D$, and categorical variables were presented as percentages. The significance of differences between the groups was assessed using Student's $t$ test for data with normal distribution, and the Mann-Whitney $\mathrm{U}$ test was used for data that were not normally distributed. For categorical variables, $\chi^{2}$ test or Fisher's exact test was used, as appropriate. Univariate and multiple Cox regression analyses were used to assess the association between device-detected AF and thromboembolic risk. Survival was estimated by the KaplanMeier method, and differences in survival between groups were assessed by the log-rank test. Statistical analysis was performed using JMP 10 (SAS Institute, Cary, North Carolina, USA). p Values of $<0.05$ were considered statistically significant.

\section{RESULTS}

\section{Detection of AHREs}

The study follow-up period was $52 \pm 30$ months (median, 48 months) with continuous cardiac rhythm monitoring between the first pacemaker implantation and the last device check or thromboembolic event. During the follow-up period, at least one AHRE occurred in 165 patients (48\%), and progression to persistent AF was observed in 36 patients (10\%) (figure 1). Baseline characteristics of the overall cohort as well as of those with and without AHREs are shown in table 1. Mean age and $\mathrm{CHADS}_{2}$ score of the overall cohort were 80 years and 2.3, respectively, which suggested the relatively high-risk of stroke for the entire study cohort. Patients with AHREs had significantly larger left atrial diameters and higher $\mathrm{CHADS}_{2}$ scores than those without AHREs. Additionally, patients with AHREs were more likely to have prior documented paroxysmal AF episodes and higher rates of anticoagulant and antiarrhythmic drug use compared with patients without AHREs. Progression to persistent AF was observed more frequently in patients with prior documented PAF than in those without (24/82 patients (29\%) vs $12 / 261$ patients $(5 \%), \mathrm{p}<0.01)$

\section{Stroke and systemic embolic events}

In the overall study population, 19 of 343 patients (6\%) experienced ischaemic stroke/SE (18 and 1 patient, respectively) during the follow-up period. The detailed characteristics of patients who suffered ischaemic stroke or SE are shown in table 2. In this group, 7 of 19 (37\%) patients were male, and the mean age was 79 years. The mean $\mathrm{CHADS}_{2}$ score of patients with ischaemic stroke/SE was significantly higher than that in patients without ischaemic stroke/SE $(3.5 \pm 1.1$ vs $2.2 \pm 1.1, \mathrm{p}<0.01)$. Fourteen patients $(74 \%)$ with ischaemic stroke/SE had at least one AHRE during the follow-up. Among these patients, AHREs within 30 days before ischaemic stroke/SE was detected in eight patients (42\%). Specifically, two patients had paroxysmal AF, whereas the remaining six patients progressed from paroxysmal to persistent AF.

\section{Associations of thromboembolic events with AHREs}

This study revealed that ischaemic stroke/SE was significantly associated with several factors (table 3). AHREs were significantly associated with ischaemic stroke/SE (HR 2.87; 95\% CI 1.10 to $8.90 ; \mathrm{p}=0.03$ ), and the Kaplan-Meier analysis demonstrated that the incidence of ischaemic stroke/SE was significantly higher in patients with AHREs than in those without AHREs $(p=0.03$; figure 2$)$. To investigate the influence of the severity of the $\mathrm{CHADS}_{2}$ score on ischaemic stroke/SE, we performed subgroup analysis according to the $\mathrm{CHADS}_{2}$ score. Patients in the study cohort were classified into three thromboembolic risk groups: low $\left(\mathrm{CHADS}_{2}\right.$ score $\left.0 ; \mathrm{n}=17\right)$, intermediate $\left(\mathrm{CHADS}_{2}\right.$ score $1-2 ; n=200)$ and high $\left(\mathrm{CHADS}_{2}\right.$ score $\left.>2 ; n=126\right)$. The relationship between thromboembolic risk and AHREs was evaluated in each group (figure 3). There was no ischaemic stroke/ SE in the low-risk group. Only three $(2 \%)$ patients in the intermediate-risk group had ischaemic stroke/SE, and AHREs were not associated with the incidence of ischaemic stroke/SE in this group $(p=0.47)$. In contrast, $16(13 \%)$ patients had ischaemic stroke/SE in the high-risk group, and the incidence of ischaemic stroke/SE was significantly higher in patients with AHREs than 
Table 1 Baseline characteristics of patients with and without AHREs

\begin{tabular}{|c|c|c|c|c|}
\hline Variable & $\begin{array}{l}\text { Overall } \\
(n=343)\end{array}$ & $\begin{array}{l}\text { Patients without AHREs } \\
(n=178)\end{array}$ & $\begin{array}{l}\text { Patients with AHREs } \\
(n=165)\end{array}$ & p Value \\
\hline Age, years & $80 \pm 7$ & $80 \pm 7$ & $80 \pm 7$ & 0.38 \\
\hline Male sex & 167 (49) & 79 (48) & $88(49)$ & 0.77 \\
\hline $\mathrm{BMI}, \mathrm{kg} / \mathrm{m}^{2}$ & $23 \pm 4$ & $23 \pm 4$ & $23 \pm 4$ & 0.26 \\
\hline Current smoking & $32(9)$ & $15(8)$ & $17(10)$ & 0.55 \\
\hline \multicolumn{5}{|l|}{ Reason for PMI } \\
\hline Sinus node disease/AV node disease & $\begin{array}{l}147 / 196 \\
(43 / 57)\end{array}$ & $\begin{array}{l}46 / 132 \\
(26 / 74)\end{array}$ & $\begin{array}{l}101 / 64 \\
(61 / 39)\end{array}$ & $<0.01$ \\
\hline $\mathrm{CHADS}_{2}$ score & $2.3 \pm 1.1$ & $2.1 \pm 1.0$ & $2.5 \pm 1.2$ & $<0.01$ \\
\hline$(0 / 1-2 />2)$ & $\begin{array}{l}17 / 200 / 126 \\
(5 / 58 / 37)\end{array}$ & $\begin{array}{l}12 / 111 / 55 \\
(7 / 62 / 31)\end{array}$ & $\begin{array}{l}5 / 89 / 71 \\
(3 / 54 / 43)\end{array}$ & 0.03 \\
\hline \multicolumn{5}{|l|}{ Comorbidities } \\
\hline Congestive heart failure & $56(16)$ & $17(10)$ & $39(24)$ & $<0.01$ \\
\hline Hypertension & $278(81)$ & $141(79)$ & $137(83)$ & 0.37 \\
\hline Diabetes mellitus & $94(27)$ & $49(28)$ & $45(27)$ & 0.96 \\
\hline Previous stroke/SE/TIA & $52(15)$ & $21(12)$ & $31(19)$ & 0.07 \\
\hline Dyslipidaemia & $136(40)$ & $72(40)$ & $64(39)$ & 0.75 \\
\hline Chronic kidney disease & $205(60)$ & $108(61)$ & $97(59)$ & 0.72 \\
\hline Coronary artery disease & $38(11)$ & $15(8)$ & $23(14)$ & 0.10 \\
\hline Cardiomyopathy & $18(5)$ & $5(3)$ & $13(8)$ & 0.04 \\
\hline Valvular heart disease & $35(10)$ & $14(8)$ & $21(8)$ & 0.14 \\
\hline Mitral valve disease & $14(4)$ & $5(3)$ & $9(5)$ & 0.22 \\
\hline Prior documented paroxysmal AF & $82(24)$ & $13(7)$ & $69(42)$ & $<0.01$ \\
\hline \multicolumn{5}{|l|}{ Echocardiography } \\
\hline LVEF, \% & $67 \pm 9$ & $67 \pm 8$ & $66 \pm 9$ & 0.31 \\
\hline $\mathrm{LAD}, \mathrm{mm}$ & $41 \pm 7$ & $40 \pm 6$ & $43 \pm 7$ & $<0.01$ \\
\hline \multicolumn{5}{|l|}{ Drug prescribed at PMI } \\
\hline Oral anticoagulant & $53(15)$ & $7(4)$ & $46(28)$ & $<0.01$ \\
\hline Antiplatelet (including aspirin) & $113(33)$ & $54(30)$ & $59(36)$ & 0.29 \\
\hline Class I or III AAD & $45(13)$ & $9(5)$ & $36(22)$ & $<0.01$ \\
\hline
\end{tabular}

Date are presented as means \pm SD or $\mathrm{n}(\%)$

$A A D$, antiarrhythmic drug; $A F$, atrial fibrillation; AHREs, atrial high rate episodes; AV node disease, atrioventricular node disease; BMI, body mass index; LAD, left atrial diameter; LVEF, left ventricular ejection fraction; PMI, pacemaker implantation; SE, systemic embolism; TIA, transient ischaemic attack.

in those without $\mathrm{AF}$ (HR 3.73; 95\% CI 1.06 to $13.1 ; \mathrm{p}=0.04$ ). However, analysis of the overall cohort revealed that the association between AHREs and ischaemic stroke/SE was not significant after adjustment for other factors such as the CHADS 2 score (HR 1.89 ; 95\% CI 0.70 to $5.98 ; \mathrm{p}=0.21$ ) and prior documented paroxysmal AF (HR 1.78; 95\% CI 0.61 to $5.94 ; \mathrm{p}=0.30$ ).

\section{DISCUSSION}

The main findings of this study were as follows. (1) During a median of 4 years of follow-up, at least one AHRE lasting for more than 6 min was observed in approximately half of the Japanese patients with pacemaker implantation included in the study. (2) AHREs were associated with ischaemic stroke/SE; however, this risk was not equal for all patients. Therefore, ischaemic stroke/SE risk should be considered particularly in patients with AHREs and high thromboembolic risk $\left(\mathrm{CHADS}_{2}\right.$ score $\left.>2\right)$.

Modern cardiac devices have sophisticated AF diagnostic algorithms that facilitate the detection of asymptomatic and brief AF episodes. ${ }^{12} 13$ Starting in the early 2000s, several studies investigated the association between device-detected AHREs and thromboembolic events. ${ }^{10}{ }^{14-16}$ Glotzer et al were the first to demonstrate that AHREs were significantly associated with stroke in the ancillary study of the MOde Selection Trial $(\mathrm{MOST})^{14}$; specifically, the authors determined that the presence of any AHREs (atrial rate $>220 \mathrm{bpm}$ for 10 consecutive beats) was an independent predictor of death or non-fatal stroke (HR 2.79).

After the MOST study, two famous prospective studies (TRENDS and ASSERT) confirmed that the presence of AHREs was associated with a two to three times increase in the risk of stroke/SE. ${ }^{10}{ }^{15}$ In agreement with the findings of these studies conducted in Western countries, this study which consisted of the Japanese population also revealed that AHREs were associated with thromboembolic events with a comparable HR of 2.87 .

Importantly, the temporal association between AHREs and stroke was examined in the subgroup analysis of the TRENDS and ASSERT. ${ }^{1718}$ Both studies described that about half of the patients who experienced stroke or SE did not have AHREs before thromboembolic events. Especially, the ASSERT investigators revealed that only 4 of 51 (8\%) patients who experienced ischaemic stroke/SE had AHREs within 30 days before these events. ${ }^{18}$ In this study, among 19 patients who experienced ischaemic stroke/SE, 14 (74\%) had AHREs before the event, whereas 8 patients (42\%) had AHREs within 30 days prior to the event. The detection rate of AHREs immediately before ischaemic stroke/SE in this study was higher than that reported in previous studies conducted in Western countries, which might be associated with the study protocols including follow-up periods. Nonetheless, in this study, as well as in previous studies, 
Table 2 Characteristics in patients with ischaemic stroke/systemic embolism

\begin{tabular}{|c|c|c|c|c|c|c|c|c|}
\hline Patient no. & Age, years & Sex & $\begin{array}{l}\text { CHADS }_{2} \\
\text { score }\end{array}$ & $\begin{array}{l}\text { Antithrombotic } \\
\text { therapy }\end{array}$ & $\begin{array}{l}\text { Prior documented } \\
\text { AF }\end{array}$ & $\begin{array}{l}\text { AHREs detected } \\
\text { during follow-up }\end{array}$ & $\begin{array}{l}\text { Progression to } \\
\text { persistent AF }\end{array}$ & $\begin{array}{l}\text { AHREs detected } \\
\leq 30 \text { days before } \\
\text { ischaemic stroke/SE }\end{array}$ \\
\hline 1 & 69 & $F$ & 2 & ASA & - & - & & \\
\hline 3 & 78 & M & 2 & None & - & - & & \\
\hline 4 & 82 & $\mathrm{~F}$ & 3 & ASA & - & - & & \\
\hline 5 & 87 & $F$ & 4 & None & - & - & & \\
\hline 7 & 75 & $\mathrm{~F}$ & 3 & ASA & - & + & - & - \\
\hline 8 & 76 & $F$ & 2 & None & + & + & - & - \\
\hline 9 & 83 & $M$ & 3 & None & - & + & - & - \\
\hline 10 & 84 & $\mathrm{~F}$ & 3 & ASA & + & + & - & - \\
\hline 11 & 87 & $\mathrm{~F}$ & 6 & None & - & + & - & - \\
\hline 12 & 67 & M & 5 & ASA & + & + & - & + \\
\hline 16 & 80 & $\mathrm{~F}$ & 5 & OAC/ASA & + & + & + & + \\
\hline 17 & 82 & $\mathrm{~F}$ & 3 & OACIASA & + & + & + & + \\
\hline 18 & 83 & M & 5 & ASA & + & + & + & + \\
\hline 19 & 87 & $\mathrm{~F}$ & 3 & None & + & + & + & + \\
\hline
\end{tabular}

$\mathrm{AF}$, atrial fibrillation; $\mathrm{AHREs}$, atrial high rate episodes; $\mathrm{ASA}$, aspirin; $\mathrm{F}$, female; $\mathrm{M}$, male; $\mathrm{OAC}$, oral anticoagulant; $\mathrm{SE}$, systemic embolism.

a substantial percentage of patients with ischaemic stroke/SE did not have any AF episodes within 30 days before the event. Therefore, the mechanism of stroke in some patients with AHREs might not be attributable to AF alone.

AHREs may play some critical roles in the development of stroke. The first is the widely supported traditional theory, which holds that AF results in cardioembolic events via atrial clots caused by mechanical stasis in the atrium. ${ }^{19-21}$ In this study, cardioembolic mechanism was strongly suspected in six patients who progressed from paroxysmal to persistent AF immediately before the ischaemic stroke/SE. In addition, Wakula et al revealed that the blood biomarkers which were associated with atrial fibrosis and progression of AF such as tissue inhibitors of metalloproteinases $-1,-2,-4$ were significantly higher in the patients with AHREs than in those without. ${ }^{22}$ These results

\begin{tabular}{|c|c|c|c|}
\hline Variable & HR & $95 \% \mathrm{Cl}$ & $\mathrm{p}$ Value \\
\hline Age & 0.99 & 0.93 to 1.07 & 0.98 \\
\hline Male & 0.68 & 0.25 to 1.69 & 0.41 \\
\hline BMI & 0.53 & 0.84 to 1.08 & 0.53 \\
\hline Current smoking & 1.03 & 0.16 to 3.62 & 0.97 \\
\hline Sinus node disease & 1.48 & 0.60 to 3.73 & 0.39 \\
\hline $\mathrm{CHADS}_{2}$ score & 2.69 & 1.83 to 3.97 & $<0.01$ \\
\hline Chronic kidney disease & 1.38 & 0.56 to 3.74 & 0.49 \\
\hline Coronary artery disease & 4.02 & 1.41 to 10.20 & 0.011 \\
\hline Cardiomyopathy & 1.94 & 0.31 to 6.80 & 0.42 \\
\hline Valvular heart disease & 2.47 & 0.70 to 6.82 & 0.14 \\
\hline LVEF & 0.98 & 0.93 to 1.07 & 0.48 \\
\hline LAD & 1.13 & 1.06 to 1.20 & $<0.01$ \\
\hline Prior documented paroxysmal AF & 4.44 & 1.78 to 11.25 & $<0.01$ \\
\hline Oral anticoagulant & 2.97 & 1.04 to 7.54 & 0.04 \\
\hline AHRES & 2.87 & 1.10 to 8.90 & 0.03 \\
\hline
\end{tabular}

atrial diameter; LVEF, left ventricular ejection fraction; SE, systemic embolism. suggest that AHREs may cause stroke because AHREs influence atrial fibrosis and the progression of AF. Alternatively, AHREs itself may become a maker of stroke in patients with cardiovascular disease. The underlying risk factors for AF (AHREs) such as $\mathrm{CHADS}_{2}$ score components (ie, age, hypertension, diabetes mellitus and heart failure) are almost similar to the risk factors for ischaemic stroke. ${ }^{23} 24$ These risk factors were shown to be associated with stroke in patients without clinical AF as they could lead to hypercoagulability and endothelial dysfunction. ${ }^{25} 26$ Therefore, AHREs may be likely to reflect thromboembolic risk. Several recent studies reported that the $\mathrm{CHADS}_{2}$ score predicted the risk for stroke in the absence of AF in patients with coronary heart disease and heart failure. ${ }^{27} 28$ In this study, AHREs were significantly associated with stroke in only the high thromboembolic risk group $\left(\mathrm{CHADS}_{2}\right.$ score $\left.>2\right)$. This result might support the hypothesis of AHREs as a maker of stroke.

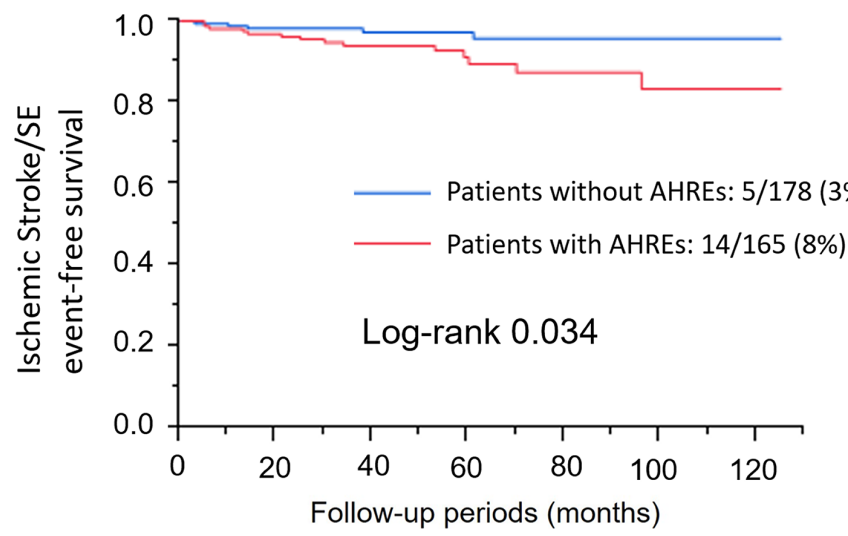

Figure 2 The Kaplan-Meier analysis demonstrating that the incidence of ischaemic stroke/systemic embolism (SE) was significantly higher in patients with atrial high-rate episodes (AHREs) than in those without AHREs. 


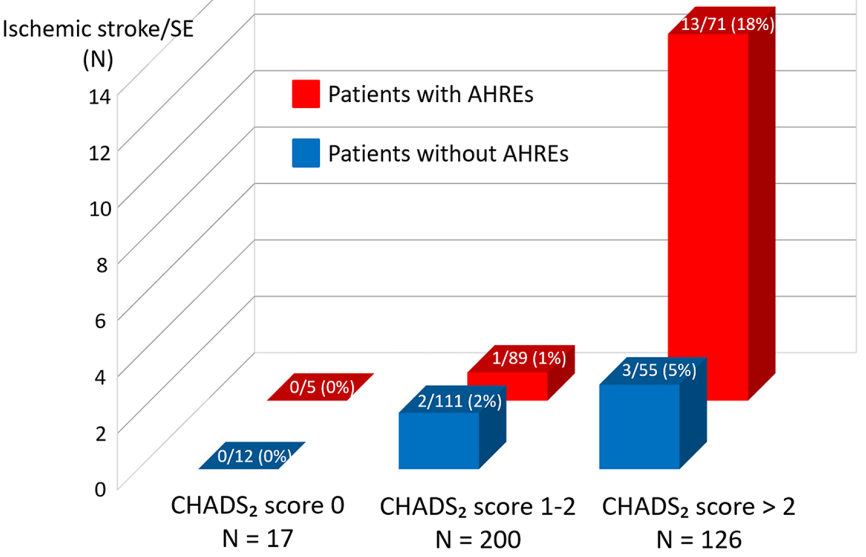

Figure 3 Association between atrial high-rate episodes (AHREs) and ischaemic stroke/systemic embolism (SE) according to the level of thromboembolic risk. The incidence of ischaemic stroke/SE was not significantly higher in patients with AHREs than in those without AHREs in either the low-risk or the intermediate-risk group. In contrast, in the high-risk group, the incidence of ischaemic stroke/SE events in patients with device-detected AHREs was significantly higher than in those without AHREs.

\section{Study limitations}

This study has several limitations. First, this was a retrospective study with a small number of patients. In particular, the number of stroke/SE is small, and only three patients experienced stroke/ $\mathrm{SE}$ events in the moderate-risk group $\left(\mathrm{CHADS}_{2}\right.$ score of $\left.1-2\right)$. It is possible that a larger study may have detected an increased risk of thromboembolic events in this population. Second, this study population included patients with previous AF, and the presence of previous AF must influence baseline antithrombotic therapy and the study outcomes. However, there were only nine ischaemic stroke/SE events in patients without previous AF (table 2); hence, statistical power was weak for the analysis of the association of AHREs with stroke/SE. There were a few previous studies which used similar study designs including prior $\mathrm{AF}^{14}{ }^{15}$; therefore, this study referred to these study designs. In addition, antithrombotic therapy was determined by the physicians attending the outpatient clinics without a uniform strategy, and we could not assess the accurate situation on the oral anticoagulant therapy including prothrombin time-international normalised ratio in the patients who were administered warfarin. Third, although modern cardiac devices have sophisticated AF diagnostic algorithms that facilitate the detection of asymptomatic and brief AF episodes, ${ }^{29}$ false positives such as repetitive non-reentrant ventriculoatrial synchrony, far-field oversensing and noise could not be completely excluded. ${ }^{30}$ We tried to confirm AHREs with intracardiac ECG in all patients, but few data of intracardiac ECG did not completely remain. Therefore, we were unable to measure AF burden details in the all the patients. Finally, we were unable to subclassify the types of stroke because most of the pacemakers used in this study were not suitable for MRI. It might be considered that few stroke events may be caused by non-thromboembolic mechanisms, including atherosclerosis, in this study. Because of these limitations, a clear demonstration of AHREs as an independent predictor of thromboembolic events was not possible in this study, which requires a much larger sample size and a longer observation period in order to collect a larger number of thromboembolic events.
Key messages

What is already known about this subject?

The relationship between atrial high-rate episodes (AHREs) detected by cardiac implantable electronic devices and thromboembolic events has been recently described in Western populations.

What does this study add?

This study revealed the clinical significance of AHREs for thromboembolic events in the Japanese population.

How might this impact on clinical practice? AHREs were associated with thromboembolic events in the Japanese population; these findings were similar to those reported in Western populations. However, this association was observed only in the high thromboembolic risk group.

\section{CONCLUSION}

AHREs were observed in approximately half of the patients and were associated with ischaemic stroke/SE in the Japanese population; these findings were similar to those reported in Western populations. However, this association was observed only in the high thromboembolic risk group.

Acknowledgements The authors thank Takashi Ishihara, Kiyomi Mukai, Keiko Furuno, Kaori Hashimoto and Mayu Ninomiya for their excellent technical assistance.

Contributors HK, TN, MS and SI planned the study and conducted a survey. FS, KN and $\mathrm{KI}$ conducted a survey. TO, TS, SU, JH and SI planned the study and revised the final manuscript. All authors approved the manuscript as submitted.

\section{Competing interests None declared.}

Patient consent Obtained.

Ethics approval The ethics committees of Ehime University Graduate School of Medicine.

Provenance and peer review Not commissioned; externally peer reviewed.

Open Access This is an Open Access article distributed in accordance with the Creative Commons Attribution Non Commercial (CC BY-NC 4.0) license, which permits others to distribute, remix, adapt, build upon this work non-commercially, and license their derivative works on different terms, provided the original work is properly cited and the use is non-commercial. See: http://creativecommons.org/ licenses/by-nc/4.0/

(C) Article author(s) (or their employer(s) unless otherwise stated in the text of the article) 2017. All rights reserved. No commercial use is permitted unless otherwise expressly granted.

\section{REFERENCES}

1 Chugh SS, Havmoeller R, Narayanan K, et al. Worldwide epidemiology of atrial fibrillation: a Global Burden of Disease 2010 Study. Circulation 2014;129:837-47.

2 Wolf PA, Abbott RD, Kannel WB. Atrial fibrillation as an independent risk factor for stroke: the Framingham Study. Stroke 1991;22:983-8.

3 January CT, Wann LS, Alpert JS, et al. AHA/ACC/HRS guideline for the management of patients with atrial fibrillation: executive summary: a report of the american college of cardiology/american heart association task force on practice guidelines and the heart rhythm society. circulation 2014;130:2071-104.

4 Kirchhof P, Benussi S, Kotecha D, et al. ESC Guidelines for the management of atrial fibrillation developed in collaboration with EACTS. Europace 2016;2016:1609-78.

5 JCS Joint Working Group. Guidelines for pharmacotherapy of atrial fibrillation (JCS 2013). Circ J 2021;2014:1997.

6 DeCicco AE, Finkel JB, Greenspon AJ, et al. Clinical significance of atrial fibrillation detected by cardiac implantable electronic devices. Heart Rhythm 2014;11:719-24.

7 Lau CP, Siu CW, Yiu KH, et al. Subclinical atrial fibrillation and stroke: insights from continuous monitoring by implanted cardiac electronic devices. Europace 2015;17(Suppl 2):ii40-ii46.

8 Gage BF, Waterman AD, Shannon W, et al. Validation of clinical classification schemes for predicting stroke: results from the National Registry of Atrial Fibrillation. JAMA 2001;285:2864-70. 
9 JCS Joint Working Group. Guidelines for non-pharmacotherapy of cardiac arrhythmias. Circ J 2013;77:249-74.

10 Healey JS, Connolly SJ, Gold MR, et al. Subclinical atrial fibrillation and the risk of stroke. N Engl J Med 2012;366:120-9.

11 Calkins H, Kuck KH, Cappato R, et al. HRS/EHRA/ECAS expert consensus statement on catheter and surgical ablation of atrial fibrillation: Recommendations for patient selection, procedural techniques, patient management and follow-up, definitions, endpoints, and research trial design: a report of the HRS task force on catheter and surgical ablation of atrial fibrillation. Developed in partnership with the EHRA, a registered branch of the ESC and the ECAS; and in collaboration with the ACC, the AHA, the APHRS, and the STS. Endorsed by the governing bodies of the ACCF, the AHA, the ECAS, the EHRA, the STS, the APHRS, and the HRS. Heart Rhythm 2012;9:632-96.

12 Pollak WM, Simmons JD, Interian A, et al. Clinical utility of intraatrial pacemaker stored electrograms to diagnose atrial fibrillation and flutter. Pacing Clin Electrophysiol 2001;24:424-9.

13 Purerfellner H, Gillis AM, Holbrook R, et al. Accuracy of atrial tachyarrhythmia detection in implantable devices with arrhythmia therapies. Pacing Clin Electrophysiol 2004:27:983-92.

14 Glotzer TV, Hellkamp AS, Zimmerman J, et al. Atrial high rate episodes detected by pacemaker diagnostics predict death and stroke: report of the atrial diagnostics ancillary study of the MOde Selection Trial (MOST). Circulation 2003;107:1614-9.

15 Glotzer TV, Daoud EG, Wyse DG, et al. The relationship between daily atrial tachyarrhythmia burden from implantable device diagnostics and stroke risk: the TRENDS study. Circ Arrhythm Electrophysiol 2009;2:474-80.

16 Boriani G, Glotzer TV, Santini M, et al. Device-detected atrial fibrillation and risk for stroke: an analysis of $>10,000$ patients from the SOS AF project (Stroke preventiOn Strategies based on Atrial Fibrillation information from implanted devices). Eur Heart J 2014;35:508-16.

17 Daoud EG, Glotzer TV, Wyse DG, et al. Temporal relationship of atrial tachyarrhythmias, cerebrovascular events, and systemic emboli based on stored device data: a subgroup analysis of TRENDS. Heart Rhythm 2011;8:1416-23.

18 Brambatti M, Connolly SJ, Gold MR, et al. Temporal relationship between subclinical atrial fibrillation and embolic events. Circulation 2014;129:2094-9.
19 Freestone B, Lip GY. The endothelium and atrial fibrillation. The prothrombotic state revisited. Hamostaseologie 2008;28:207-12.

20 Watson T, Shantsila E, Lip GY. Mechanisms of thrombogenesis in atrial fibrillation: virchow's triad revisited. Lancet 2009;373:155-66.

21 Guo Y, Lip GY, Apostolakis S. Inflammation in atrial fibrillation. J Am Coll Cardiol 2012;60:2263-70

22. Wakula P, Neumann B, Kienemund J, et al. CHA2DS2-VASc score and blood biomarkers to identify patients with atrial high-rate episodes and paroxysmal atrial fibrillation. Europace 2017;19:544-51.

23 Kirchhof P, Lip GY, Van Gelder IC, et al. Comprehensive risk reduction in patients with atrial fibrillation: emerging diagnostic and therapeutic options--a report from the 3rd Atrial Fibrillation Competence NETwork/European Heart Rhythm Association consensus conference. Europace 2012;14:8-27.

24 Ohira T, Shahar E, Chambless LE, et al. Risk factors for ischemic stroke subtypes: the atherosclerosis risk in communities study. Stroke 2006;37:2493-8.

25 McClung JA, Naseer N, Saleem M, et al. Circulating endothelial cells are elevated in patients with type 2 diabetes mellitus independently of $\left.\mathrm{HbA}_{(1)}\right) \mathrm{c}$. Diabetologia 2005:48:345-50.

26 Haeusler KG, Laufs U, Endres M. Chronic heart failure and ischemic stroke. Stroke 2011:42:2977-82.

27 Welles CC, Whooley MA, Na B, et al. The CHADS2 score predicts ischemic stroke in the absence of atrial fibrillation among subjects with coronary heart disease: data from the heart and soul study. Am Heart J 2011;162:555-61.

28 Mitchell LB, Southern DA, Galbraith D, et al. Prediction of stroke or TIA in patients without atrial fibrillation using CHADS2 and CHA2DS2-VASC scores. Heart 2014; 100:1524-30.

29 Pollak WM, Simmons JD, Interian A, et al. Clinical utility of intraatrial pacemaker stored electrograms to diagnose atrial fibrillation and flutter. Pacing Clin Electrophysiol 2001;24:424-9.

30 Kaufman ES, Israel CW, Nair GM, et al. Positive predictive value of device-detected atrial high-rate episodes at different rates and durations: an analysis from ASSERT. Heart Rhythm 2012;9:1241-6. 